\title{
Veranstaltungen auch in Zeiten von Corona
}

\section{Die Videoreihe der Bibliothek für Zeitgeschichte}

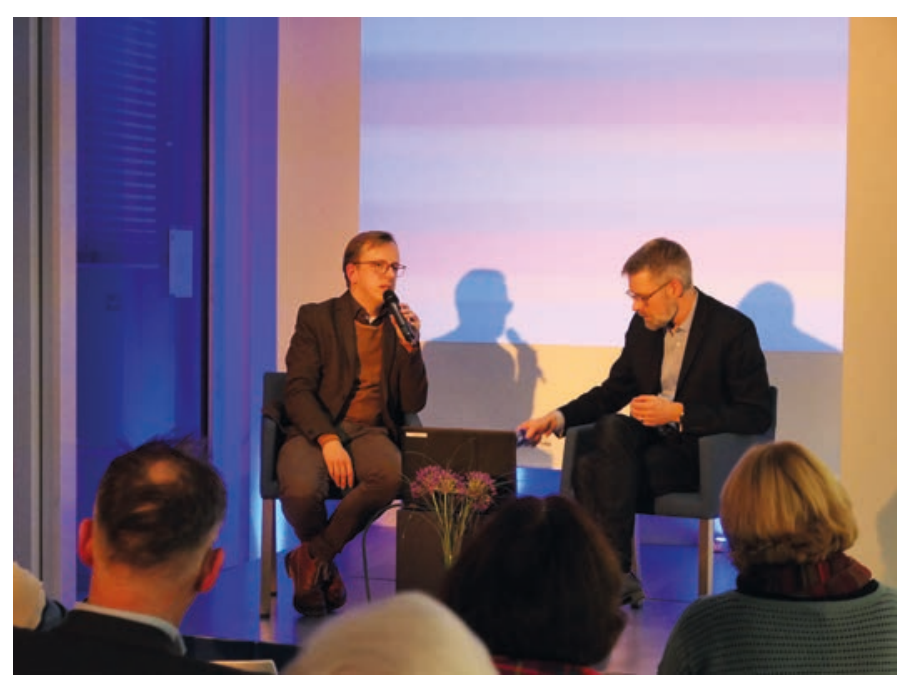

Abb. 1: Marcus Böick (links), erster Referent der Videoreihe der BfZ, sprach im Januar 2020 über die Treuhand.

2020, das als Corona-Jahr in die Geschichtsbücher eingehen wird, machte allen Organisatoren von kulturellen und wissenschaftlichen Veranstaltungen das Leben schwer. Die Abfolge von Shutdowns und partiellen Wiedereröffnungen unter Pandemie-Bedingungen zwang die Veranstalter, Termine immer wieder umzuplanen - oder in die digitale Welt zu wechseln. Dieser Herausforderung stellte sich auch die Bibliothek für Zeitgeschichte (BfZ), die im Jahr zehn Veranstaltungen zu Politik und Zeitgeschehen anbietet.

Es erwies sich in dieser Situation als überaus glückliche Fügung, dass die BfZ im Januar 2020 begonnen hatte, ihre Vortragsreihe in Kooperation mit der Gerda Henkel Stiftung aufzuzeichnen. Bereits 2019, als Covid-19 noch ein weit entferntes Problem zu sein schien, trat die Düsseldorfer Stiftung, die sich der Förderung der historischen Geisteswissenschaften verschrieben hat, an die BfZ heran. Sie bot an, die technische Ausstattung für Mitschnitte der Vorträge, Lesungen und Gespräche der BfZ bereitzustellen und die Videos auf dem Wissenschaftsportal L.I.S.A. ${ }^{1}$ zu veröffentlichen. Dort sollten sie als Video und Audiomitschnitt zur Verfügung gestellt werden. Die BfZ nahm dieses bestechende Angebot dankend an und machte sich an die Umsetzung.
Zunächst ging es darum, Präsenz-Veranstaltungen zeitversetzt auch im Internet zu zeigen und damit über Stuttgart hinaus jederzeit und dauerhaft einer breiten Öffentlichkeit zugänglich zu machen. Das Portal L.I.S.A. bietet ein breites Spektrum an Interviews, Diskussionen, Vorträgen, Dokumentationen und Reportagen zu historischen Themen und geschichtswissenschaftlichen Debatten. Hier reihte sich die Vortragsreihe der BfZ mit ihren ersten "digitalen" Referenten Marcus Böick und Benjamin Ziemann perfekt ein. Die Vorträge zur Treuhand² und zu Martin Niemöller ${ }^{3}$ wurden bis heute mehr als tausendmal aufgerufen, Ziemanns Beitrag sogar fast zweitausendmal.

Während des ersten Shutdowns von März bis Mai 2020 eröffnete sich dann die Chance, mit rein digitalen Formaten einen Ersatz für die ausfallenden Präsenz-Veranstaltungen zu schaffen. So entstanden in der BfZ Aufnahmen mit Gerhard Hirschfeld zum Kriegsende $1945^{4}$ und mit Martin Cüppers zu einem Fotoalbum von KZ-Kommandant Johann Niemann, ${ }^{5}$ einer wichtigen Bildquelle zum Holocaust. Da sowohl der ehemalige Direktor der BfZ als auch der wissenschaftliche Leiter der Forschungsstelle Ludwigsburg keinen weiten Anreiseweg hatten, konnte mit ihnen vor Ort eine Lesung bzw. ein Gespräch realisiert werden.

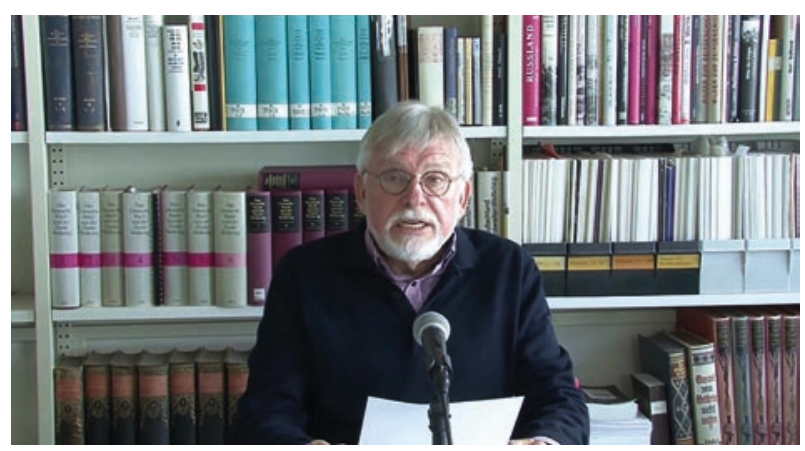

Abb. 2: Gerhard Hirschfeld bestritt im Mai 2020 mit seiner Lesung zum Kriegsende 1945 die erste rein digitale Veranstaltung der BfZ.
1) https://lisa.gerda-henkel-stiftung.de/dossier_bfz_stuttgart

2) https://lisa.gerda-henkel-stiftung.de/marcus_boeick_die_treuhand_ idee_praxis_erfahrung 1990_1994?nav id=8877

3) https://lisa.gerda-henkel-stiftung.de/benjamin_ziemann_martin_niemoeller_ein_leben_in_opposition?nav_id=8906

4) https://lisa.gerda-henkel-stiftung.de/lesung_8mai_hirschfeld

5) https://lisa.gerda-henkel-stiftung.de/bfz_fotos_aus_sobibor 
Die Lockerung der Bestimmungen zur Pandemiebekämpfung machte dann im Juli wieder PräsenzVeranstaltungen möglich, allerdings mit deutlich reduzierter Teilnehmerzahl. Die neue Lage wurde genutzt, um mit Peter Walther eine Lesung mit Gespräch zum Ende der Weimarer Republik ${ }^{6}$ abzuhalten. Am 22. Oktober 2020 fand dann die erste BfZ-Veranstaltung im neuen Vortragsraum der WLB statt: Christian Westerhoff diskutierte mit Julia von Staden und Philipp Gassert über die bunte und lebendige Protestkultur, die sich in den letzten Jahren unter anderem in Stuttgart etabliert hat. ${ }^{\text {? }}$

Doch die Freude über die Möglichkeit, wieder vor Publikum aufzutreten, währte nur kurz: Ab dem

2. November 2020 galt ein neuer "Lockdown light". Präsenz-Veranstaltungen rückten bis auf Weiteres wieder in weite Ferne. Die BfZ nahm dies zum Anlass, den nächsten Schritt in die digitale Welt zu nehmen: Um nicht auf Referent*innen verzichten zu müssen, die außerhalb des Großraums Stuttgart leben, wurden die folgenden Interviews mithilfe einer Videokonferenz-Software durchgeführt. Diese Software ermöglicht es, ein Gespräch mit mehreren Personen aufzunehmen, die sich an unterschiedlichen Orten aufhalten. Dies sorgt nicht nur für eine große Flexibilität, sondern es erspart den Referent*innen auch die Anreise und zahlreiche Kontakte - ein Argument, das mit den erneut stark steigenden Corona-Zahlen im Laufe der Wintermonate immer wichtiger wurde.

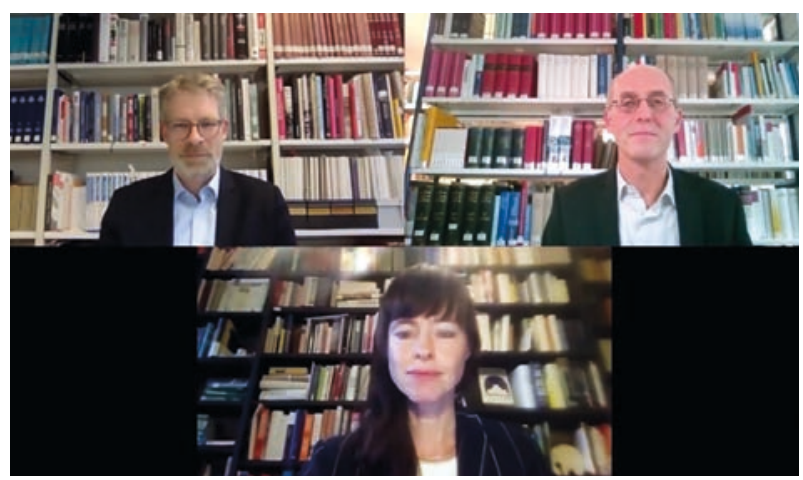

Abb. 3: Hedwig Richter stellte im Dezember 2020 in einem Video-Gespräch ihr Buch "Demokratie: Eine deutsche Affäre. Vom 18. Jahrhundert bis zur Gegenwart" vor.

Mittlerweile stehen mehrere Videos zur Verfügung, die auf diese Weise entstanden sind. Noch im Dezember 2020 stellte Hedwig Richter ihr viel diskutiertes Buch „Demokratie: Eine deutsche Affäre. Vom 18. Jahrhundert bis zur Gegenwart" vor. ${ }^{8}$ Damit fand ein schwieriges Jahr einen erfolgreichen Abschluss.

Der Sprung in die digitale Veranstaltungs-Welt ist also gelungen. Dennoch hofft die BfZ, bald auch wieder Präsenz-Veranstaltungen durchführen zu können. Zwar ist es sehr erfreulich, wenn man sich einen Vortrag oder eine Diskussion im Internet ansehen oder anhören kann, z.B. weil man in einer anderen Stadt wohnt oder aus zeitlichen Gründen keine Gelegenheit hatte, an einem Termin teilzunehmen. Doch der Austausch und die persönlichen Kontakte, die eine Live-Veranstaltung mit sich bringen, sind nun einmal nicht zu ersetzen. 